UCRL-2495

Unclassified Instrumentation Distribution

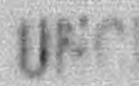

UNIVERSITY OF CALIFORNIA

Radiation Leboratory

Contract No. W-7405-eng -48

A LINEAR, TRANSPARENT BFAM INTEGRATOR

Sumner $W$. Kitchen

February 12, 1954

Berkeley, California 
Unclassified Instrumentation Distribution

\title{
A LINEAR, TRANSPARENT BEAM INTEGRATOR
}

Surnner $W$. Kitchen

Radiation Laboratory, Department of Physics, University of California, Berkeley. California

February 12, 1954

\begin{abstract}
A fast, linear air-ionization chamber has been developed for integrating and monitoring external heavy-particle beams. It consists of a thin-windowed air chamber in which the beam ionization light is viewed by a photomultiplier whose output is fed directly to a standard electrometer.
\end{abstract}




\title{
A LINEAR, TRANSPARENT BEAM INTEGRATOR
}

\author{
Sumner $W$. Kitchen
}

Radiation Laboratory. Department of Physice. University of California, Berkeley, California

February 12, 1954

For determining the excitation curve of a short-lived nuclear reaction with an external particle beam, the common methode for measuring the integrated beam actually incident on the target - - such as a Faraday cup or an ionization chamber --fail for one or more of the following reasons: (1) the time delay between the end of bombardment and the start of counting is too great: (2) an increase in absorber scatters a greater proportion of the beam out of the integrator: (3) the integrator is nonlinear with changing beam current or beam energy. The integrator described below, however, permits an accurate measurement of the relative amount of beam incident on the target.

In essence, the integrator consists of an air ionization chamber viewed by a photomultiplier whose output is fed into a standard integrating electrometer and recorder. When compared to a Faraday cup integrator* using from $\sim 10^{-10}$ to $10^{-7}$ amperes from the Berkeley linear accelerator, this syotem proved to be linear, within $\pm 1 \%$, within the limits set by the electrometer and the dark current. The particular electrometer used became nonlinear when the instantaneous current from the photomultiplier exceeded one milliampere, well below the limit of nonlinearity of the DuMont 6291 photomultiplier. The measured dark current, on the other hand, was only $2 \times 10^{-9}$ amperes. This low dark current was achieved by choice of phototube type and by gating the second dynode on only during the beam pulse, as shown in Fig. 1 (a).

Despite the inherently long range of linearity of the 6291 , the output of the photomultiplier integrator was not linear with beam current until the condensers on the last three dynodes were increased by a factor of about 10 to their present values and the divider string was reduced from I megohm to 0.270 megohm. In practice, it was more important that the capacity per stage be large enough to hold the potential of the dynode within a fraction of a volt during a pulse than that the RC time constant be less than the interval between pulses.

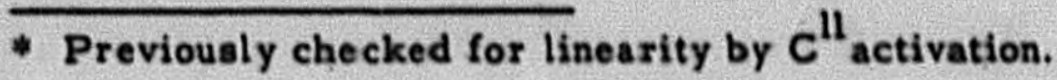


The success of the photomultiplier integrator suggested a solution to another problem associated with boinbardments of this kind at the linear accelerator, namely, monitoring the beam current. The nontinearities of conventional ionization chambers and fadiation meters make it difficult for an operator to keep the beam optimized. By taking the signal from the last dynode rather than the anode, as shown in Fig. 1 (b), the photomultiplier can be coupled to the standard curreat-monitor electrometer, thus providing a linear current monitor simultaneously with integration.

This method of current-monitoring provides a second signal free, vis., a beam pulse -monitoring signal from the anode, Fig. 2 (a) is a picture of the normal pulse shape, whereas Fig 2 (b) shows the effect of low $\mathrm{rf}$ voltage in the accelerator. Aberrations in the Van de Graaff or the source adjustments exhibit similar characteristic distortions of the pulse shape.

Two phototubes, together with a suitable gating circuit, are required to fulfill all the above functions. The unit presently in use at the linear accelerator is shown in Fig. 3. Despite the presence of a quadrupole magnetic lens near by and the high radiation background during bombardment, no shielding other than black masking tape was found necessary.

The one drawback to this integrator is that it must be calibrated, since it is placed between the accelerator and the absorbers. Prior to a bombardment, therefore, three steps are taken: (1) the photomultiplier voltage is turned up until the peak current out of the photomultiplier for maximum beam is one milliampere, which occurs normally at about 750 volts: (2) the solid angle of the target is defined by a suitable mask in front of a Faraday cup: (3) the ratio of the charge collected from the cup to that from the photomultiplier is then measured as a function of absorber thickness.

The author wishes to thank J.D. Gow and R. E. Thomas for fruitful discussions during the evolution of the integrator into its present form, F. E. Vogeleberg for the gating circuit, and the crew of the tinear accelerator for their fine cooperation.

This work was done under the auspices of the Atomic Energy Commisaion. 
$-5$.

UCRL*2495

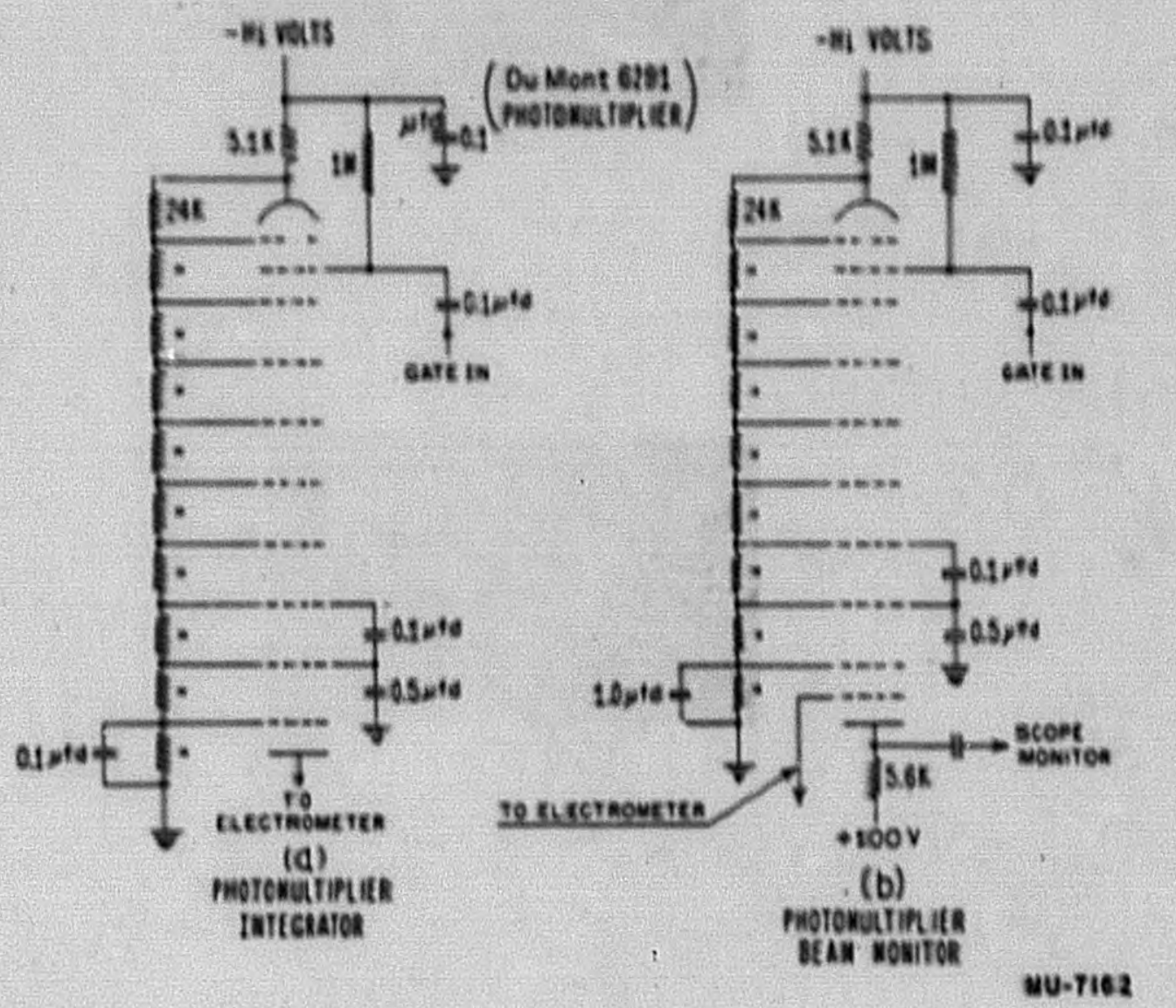

Fig. I 
UCRL -249"

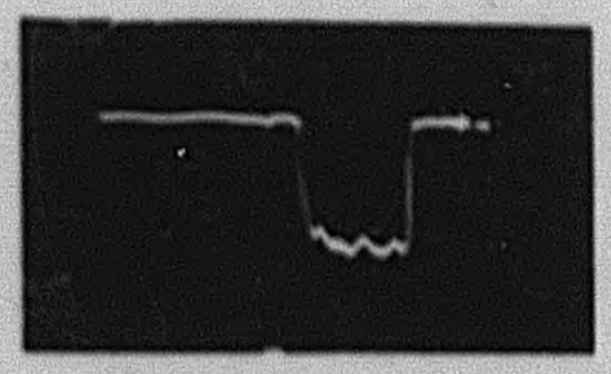

(a)

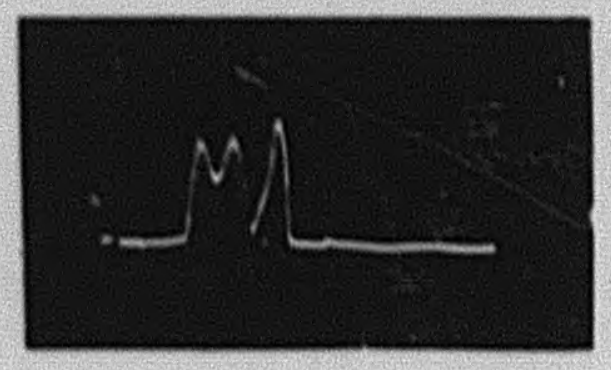

(b)

ZN-908

Fig. 2 


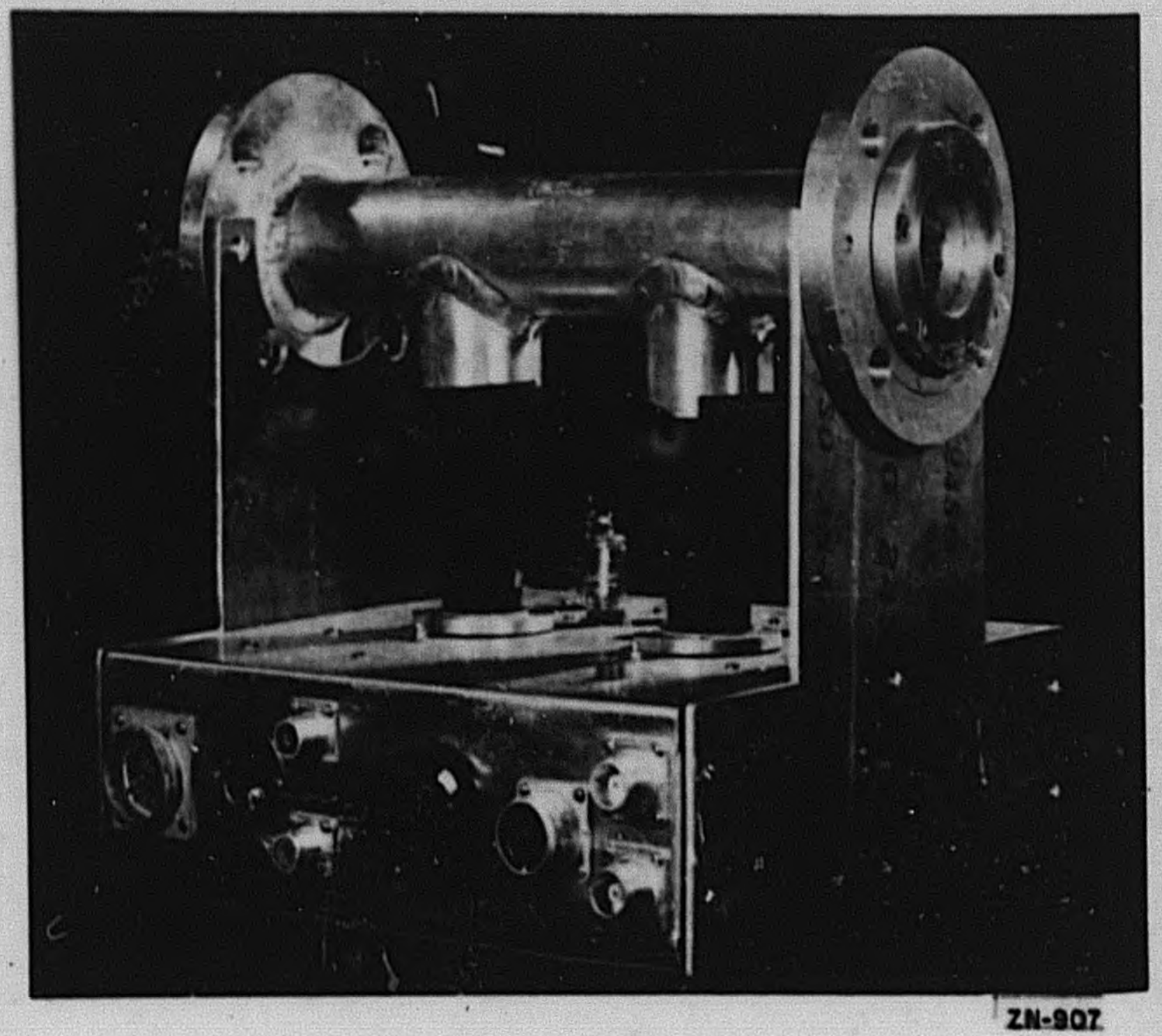

Fig. 3

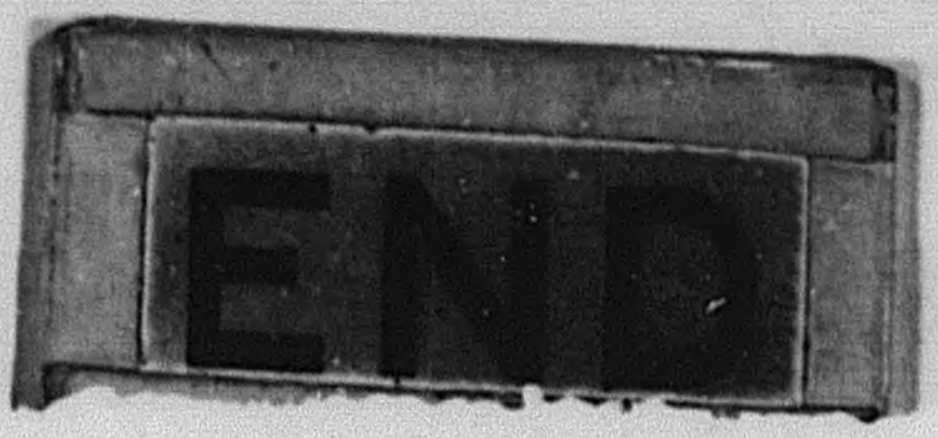

